\title{
Recombination of Point Defects via Extended Defects and Its Influence on Dopant Diffusion
}

\author{
D. Sticbel, P. Pichler \\ Fraunhofer-Institut für Integrierte Schaltungen, Bauelementetechnologic, \\ Schottkystrasse 10, 91058 Erlangen, Germany
}

\begin{abstract}
The transient enhanced diffusion of dopants in silicon is known to be governed by the interaction of extended defects and intrinsic point defects. In this work we present calculations of the formation of extended defects and their interactions with point defects, based on $a b$-initio calculations of Gilmer, Caturla, and coworkers. Dissolution of the extended defects may occur either by diffusion of point defects to surfaces and interfaces, or by reactions in the bulk. The work presented here emphasizes especially the reaction of point defects with extended defects which is shown to be more effective than bulk recombination.
\end{abstract}

\section{Introduction}

Modeling of transient enhanced diffusion has changed from a point-defect-centered view to the understanding that the storage of point defects in extended defects is the main mechanism determining the kinetics of the phenomenon [1]. Extended defects form by agglomeration of intrinsic point defects generated by the ion implantation process. Agglomeration occurs already at room temperature, but is accelerated at elevated temperatures. During annealing, Ostwald ripening of the extended defects will lead to a growth of the large ones on the expense of smaller extended defects. In addition, they will dissolve and maintain point-defect concentrations which exceed their equilibrium value and promote enhanced diffusion of dopants.

Various processes may contribute to the reduction of the total number of point defects, i. e., free point defects and point defects in clusters. Besides diffusion to and individual recombination of point defects at surfaces, one has to consider especially bulk recombination, the mutual annihilation of self-interstitials and vacancies, and "cluster recombination", the reaction of point defects with point-defect clusters. It will be shown that, as soon as the majority of the point defects has agglomerated, cluster recombination is much more efficient than bulk recombination.

\section{The Physical Model}

In this work, the description of the redistribution of intrinsic point defects and their interaction with extended defects is based on a diffusion-reaction scheme. The system of equations to be solved contains continuity equations for each of the point defects involved, as well as for each size of the point-defect clusters. For the point-defect clusters, sizes of up. to 40 point defects per cluster were taken into account. By increasing this number for comparison, it was ascertained that this maximum number 
had no influence on the results. The continuity equations are coupled by terms which describe the reactions between the respective defects. In particular, growth and shrinkage of, e. g., self-interstitial clusters $I_{n}$ of size $n$ was considered to proceed either via incorporation or release of an additional self-interstitial $I$ via

$$
I_{n}+I \nRightarrow I_{n+1}
$$

or via an interaction with vacancies $V$ in the form

$$
I_{n}+V \rightleftharpoons I_{n-1}
$$

The simulations are based on point-defect parameters proposed by Sinno and Brown [2]. Clustering and recombination reactions are included by using the assumption of a diffusion limited process. The dependence of the capture radius on the size of the extended defects used reflects the assumption of a spherical shape. But this particular choice has an insignificant influence on the conclusions. The backward rates of the cluster-growth reactions (1) are expressed in terms of the binding energies taken from $a b$-initio calculations $[3,4,5]$. For large clusters, the binding energies are given by $E_{n}^{V}=3.6 \mathrm{eV}-4.9 \mathrm{eV} \cdot\left((n+1)^{2 / 3}-n^{2 / 3}\right)$ and $E_{n}^{I}=2.5 \mathrm{eV}-2.17 \mathrm{eV} \cdot((n+$ $\left.1)^{1 / 2}-n^{1 / 2}\right)$. Backward reaction rates for bulk recombination and for the reaction of vacancy clusters with self-interstitials and vice versa (2) can be computed from detailed equilibrium. Table 1 summarizes the reaction rates used.

\begin{tabular}{|l|l|l|}
\hline Reaction & Forward reaction rate & Backward reaction rate \\
\hline \hline$I+V \rightleftharpoons 0$ & $4 \pi \cdot 0.235 \mathrm{~nm} \cdot\left(D_{I}+D_{V}\right)$ & follows from detailed equilibrium \\
\hline \hline$+I \rightleftharpoons I_{2}$ & $8 \pi \cdot 0.235 \mathrm{~nm} \cdot D_{I}$ & $D_{I} \cdot \exp (-1.60 \mathrm{eV} / k T) / a^{2}$ \\
\hline$I+I_{2} \rightleftharpoons I_{3}$ & $4 \pi \cdot 0.296 \mathrm{~nm} \cdot D_{I}$ & $D_{I} \cdot \exp (-2.25 \mathrm{eV} / k T) / a^{2}$ \\
\hline$I+I_{3} \rightleftharpoons I_{4}$ & $4 \pi \cdot 0.339 \mathrm{~nm} \cdot D_{I}$ & $D_{I} \cdot \exp (-1.29 \mathrm{eV} / k T) / a^{2}$ \\
\hline$I+I_{4} \rightleftharpoons I_{5}$ & $4 \pi \cdot 0.373 \mathrm{~nm} \cdot D_{I}$ & $D_{I} \cdot \exp (-2.29 \mathrm{eV} / k T) / a^{2}$ \\
\hline$I+I_{n} \rightleftharpoons I_{n+1}$ & $4 \pi \cdot n^{1 / 3} \cdot 0.235 \mathrm{~nm} \cdot D_{I}$ & $D_{I} \cdot \exp \left(-E_{n}^{I} / k T\right) / a^{2}$ \\
\hline \hline$V+V \rightleftharpoons V_{2}$ & $8 \pi \cdot 0.235 \mathrm{~nm} \cdot D_{V}$ & $D_{V} \cdot \exp (-0.62 \mathrm{eV} / k T) / a^{2}$ \\
\hline$V+V_{2} \rightleftharpoons V_{3}$ & $4 \pi \cdot 0.296 \mathrm{~nm} \cdot D_{V}$ & $D_{V} \cdot \exp (-0.78 \mathrm{eV} / k T) / a^{2}$ \\
\hline$V+V_{3} \rightleftharpoons V_{4}$ & $4 \pi \cdot 0.339 \mathrm{~nm} \cdot D_{V}$ & $D_{V} \cdot \exp (-1.20 \mathrm{eV} / k T) / a^{2}$ \\
\hline$V+V_{4} \rightleftharpoons V_{5}$ & $4 \pi \cdot 0.373 \mathrm{~nm} \cdot D_{V}$ & $D_{V} \cdot \exp (-1.82 \mathrm{eV} / k T) / a^{2}$ \\
\hline$V+V_{n} \rightleftharpoons V_{n+1}$ & $4 \pi \cdot n^{1 / 3} \cdot 0.235 \mathrm{~nm} \cdot D_{V}$ & $D_{V} \cdot \exp \left(-E_{n}^{V} / k T\right) / a^{2}$ \\
\hline \hline$I_{n}+V \rightleftharpoons I_{n-1}$ & $4 \pi \cdot n^{1 / 3} \cdot 0.235 \mathrm{~nm} \cdot D_{V}$ & follows from detailed equilibrium \\
\hline$V_{n}+I \rightleftharpoons V_{n-1}$ & $4 \pi \cdot n^{1 / 3} \cdot 0.235 \mathrm{~nm} \cdot D_{I}$ & follows from detailed equilibrium \\
\hline
\end{tabular}

Table 1: Reaction rates as a function of the diffusion coefficients $D_{I}$ and $D_{V}$ of self-interstitials and vacancies. The constant $a$ stands for the inter-atomic spacing.

\section{Results and Discussion}

In order to investigate the time dependence of the defect concentrations, the system of coupled continuity equations outlined above was solved numerically. All examples discussed below were obtained for a nominal process temperature of $850^{\circ} \mathrm{C}$. The initial concentrations of the intrinsic point defects were set to a value of $10^{20} \mathrm{~cm}^{-3}$. 

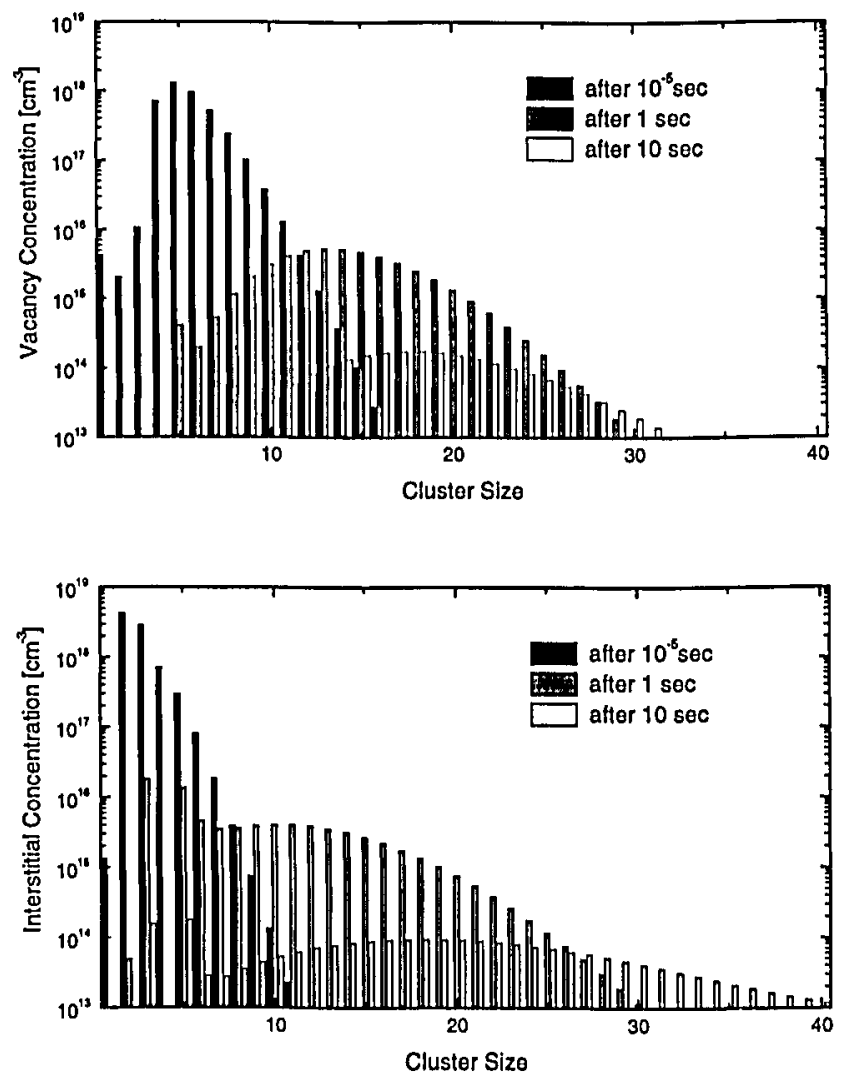

Figure 1: Distribution of vacancies and interstitials among the clusters at different process times.

Fig. 1 shows snapshots of histograms of the concentrations of vacancies and selfinterstitials in clusters. It is apparent that the point-defect clusters undergo a process of Ostwald ripening. When surface recombination is negligible, as it was assumed here for the sake of simplicity, bulk recombination and cluster recombination are the only effective mechanisms for the reduction of the total number of point defects. In Fig. 2, the effects of various assumptions on the reaction mechanisms are compared. Recombination between free point defects is very effective when clustering is ignored. When clustering is included, the free point defects are thereby reduced so rapidly that bulk recombination becomes ineffective, and the total concentration of point defects remains nearly constant for times exceeding $10^{-6}$ seconds. When cluster recombination is assumed in addition, a steady decrease of the total concentration of point defects will result. This decrease is related to the rather high concentration of point-defect clusters in comparison to the concentrations of the point defects. As a consequence, the transient-enhanced diffusion of dopants will decrease on a much shorter timescale compared with the situation neglecting cluster recombination.

Simulations based on the point-defect parameters calculated by Gilmer [3] resulted in very similar results. This suggests that the results are not very sensitive on point- 


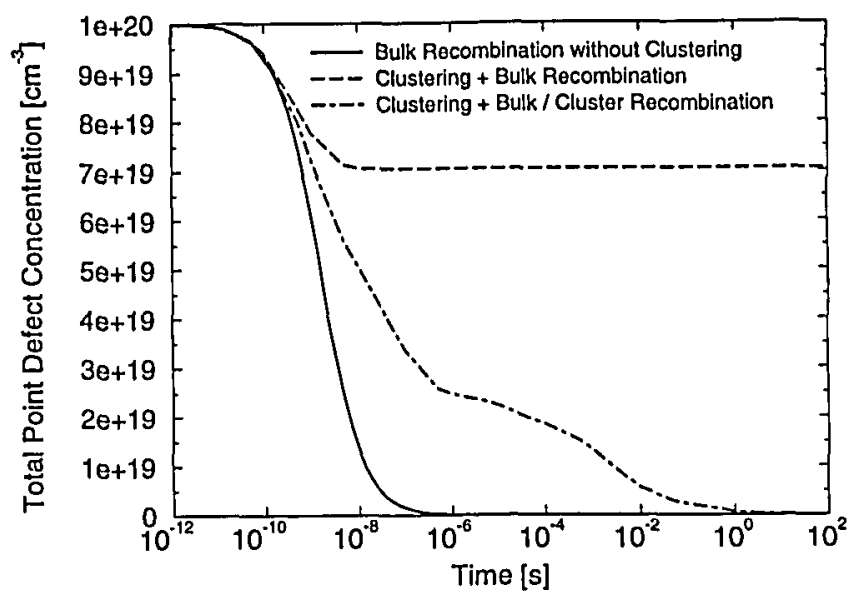

Figure 2: Total point-defect concentration as a function of time

defect parameters as long as the respective products of equilibrium concentration and diffusion coefficient are comparable.

\section{Conclusions}

Although being just a first conclusion of this work, we note that reactions of selfinterstitials with vacancy clusters and of vacancies with self-interstitial clusters are indispensable for a quantitative simulation of transient-enhanced diffusion.

\section{Acknowledgements}

The authors want to thank Dr. Caturla for the very fruitful discussions. This work was part of the project RAPID, funded in part as ESPRIT Long Term Research project No. 23481.

\section{References}

[1] D.J. Eaglesham, P.A. Stolk, H.-J. Gossmann and J.M. Poate, "Implantation and Transient B Diffusion in Si: The Source of the Interstitials", Appl. Phys. Lett., vol. 65, no. 18, pp. 2305-2307, 1994

[2] T. Sinno, R.A. Brown, W. von Ammon and E. Dornberger, "On the Dynamics of the Oxidation-Induced Stacking-Fault Ring in As-Grown Czochralski Silicon Crystals", Appl. Phys. Lett., vol. 70, no. 17, pp. 2250-2252, 1997

[3] G.H. Gilmer, T. Diaz de la Rubia, D.M. Stock and M. Jaraiz, "Diffusion and Interaction of Point Defects in Silicon: Molecular Dynamics Simulations, Nucl. Instr. Meth. Pliys. Res. B, vol. 102, pp. 247-255, 1995

[4] M.-J. Caturla, T. Diaz de la Rubia, M. Jaraiz and G.H. Gilmer, "Atomic Scale Simulations of Arsenic Ion Implantation and Annealing in Silicon", Mat. Res. Soc. Proc., vol. 396, pp. 45-50, 1996

[5] M.-J. Caturla (private communication) 